\title{
Intranasal oxytocin enhances socially-reinforced learning in rhesus monkeys
}

\author{
Lisa A. Parr * \\ Department of Psychiatry and Behavioral Science, Center for Translational Social Neuroscience and Yerkes National Primate Research Center, \\ Emory University, Atlanta, GA, USA
}

\section{Edited by:}

Alaine Keebaugh, Emory University, USA

\section{Reviewed by:}

René Hurlemann, University of Bonn, Germany

Alexa H. Veenema, Boston College, USA

\section{*Correspondence:}

Lisa A. Parr, Department of Psychiatry and Behavioral Science, Center for Translational Social Neuroscience and Yerkes National Primate Research Center, Emory University, 954 Gatewood Rd., Atlanta, GA 30329, USA

e-mail: Iparr@emory.edu
There are currently no drugs approved for the treatment of social deficits associated with autism spectrum disorders (ASD). One hypothesis for these deficits is that individuals with ASD lack the motivation to attend to social cues because those cues are not implicitly rewarding. Therefore, any drug that could enhance the rewarding quality of social stimuli could have a profound impact on the treatment of ASD, and other social disorders. Oxytocin (OT) is a neuropeptide that has been effective in enhancing social cognition and social reward in humans. The present study examined the ability of OT to selectively enhance learning after social compared to nonsocial reward in rhesus monkeys, an important species for modeling the neurobiology of social behavior in humans. Monkeys were required to learn an implicit visual matching task after receiving either intranasal (IN) OT or Placebo (saline). Correct trials were rewarded with the presentation of positive and negative social (play faces/threat faces) or nonsocial (banana/cage locks) stimuli, plus food. Incorrect trials were not rewarded. Results demonstrated a strong effect of sociallyreinforced learning, monkeys' performed significantly better when reinforced with social vs. nonsocial stimuli. Additionally, socially-reinforced learning was significantly better and occurred faster after IN-OT compared to placebo treatment. Performance in the IN-OT, but not Placebo, condition was also significantly better when the reinforcement stimuli were emotionally positive compared to negative facial expressions. These data support the hypothesis that OT may function to enhance prosocial behavior in primates by increasing the rewarding quality of emotionally positive, social compared to emotionally negative or nonsocial images. These data also support the use of the rhesus monkey as a model for exploring the neurobiological basis of social behavior and its impairment.

Keywords: oxytocin, social reward, learning, monkey, autism, facial expressions, emotion
Oxytocin (OT) is a neurohypophyseal peptide that acts as a neuromodulator in the brain where it plays a key role in regulating social and emotional behaviors (Neumann et al., 2013). In rodents, for example, OT is important for pair bonding and affiliation, social recognition, and alloparental care (Ross and Young, 2009). Because of these prosocial effects, the oxytocinergic system has emerged as a leading target for the development of novel pharmacotherapies for treating social impairments, like those seen in autism spectrum disorders (ASD; Guastella and MacLeod, 2012; Modi and Young, 2012). Although nonapeptides, like OT and vasopression, are large molecular weight compounds that do not readily cross the blood brain barrier when given peripherally, several studies have now confirmed the effectiveness of an intranasal (IN) administration route for delivering these compounds to the central nervous system (CNS) in humans and animals (Born et al., 2002; Chang et al., 2012; Neumann et al., 2013; Striepens et al., 2013; Modi et al., 2014). Using this method, studies have reported broad effects of OT on the perception of social stimuli including increasing the salience of the eyes in faces (Guastella et al., 2008; Andari et al., 2010), altering the perception of facial expressions (Domes et al., 2007a,b; Fischer-Shofty et al.,
2010; Gamer et al., 2010; Parr et al., 2013), enhancing memory for face identity (Savaskan et al., 2008; Rimmele et al., 2009), and decreasing social vigilance (Ebitz et al., 2013). Additionally, several studies have proposed a positive relationship between OT and social reward (Strathearn et al., 2009; Hurlemann et al., 2010). In a clever study, Hurlemann et al. (2010) examined the effects of OT on an implicit learning task when participants were reinforced with either social or nonsocial stimuli. Participants were required to categorize a series of 3-digit number strings into one of two randomly assigned categories. In the social condition, each trial began with the number string and a neutral face. After correct responses, the neutral face changed to a happy, smiling expression but after incorrect responses, the neutral face turned angry. In the nonsocial condition, the trial began with the number string and a black circle which turned green if the subject chose correctly and red if the answer was incorrect. These authors found that INOT enhanced subjects performance when reinforced with social compared to nonsocial images.

The positive relationship between OT and social reward has important implications for the development of treatments for individuals with ASD. For example, the Social Motivation Theory 
proposes that individuals with ASD fail to find social stimuli or social interactions intrinsically rewarding, thus contributing to their social impairments by reducing attention to social cues and dampening interest in social interactions (Dawson et al., 1998; Chevallier et al., 2012). Neuroimaging studies have reported reduced activation in the striatum of individuals with ASD in response to social but not nonsocial (monetary) rewards (ScottVan Zeeland et al., 2010; Delmonte et al., 2012). The striatum is a key structure in the brain's neural reward circuitry and is responsible for associating a reward value to specific behaviors. In the case of ASD, failure to develop rewarding contingencies between social stimuli and behavior could lead to impaired social learning, reduced social interest, and long-term deficits in social cognition. OT represents an exciting potential pharmacotherapy for the treatment of social impairments because any drug that could increase the salience of social cues and prosocial motivation, or enhance the rewarding value of social stimuli and interactions could significantly improve the outcome of existing therapies for individuals with ASD and related disorders. Examining social deficits in autism from the perspective of reward processing may lead to novel insights for potential treatments and interventions.

To date, several single dose studies using intranasal OT have shown promising results by improving attention to social cues and reducing repetitive behaviors in adults with ASD (Hollander et al., 2003; Andari et al., 2010; Guastella et al., 2010; Domes et al., 2013; Preti et al., 2014). Despite these encouraging results, many questions remain including where in the brain OT is exerting its prosocial effects, its mechanism of action, the best way to administer peptides to young children, effects of repeated treatments, and the range of therapeutic doses (Churchland and Winkielman, 2012). Animal models are critically needed to address these questions. Monkeys represent an ideal species for this purpose because the distribution of OT receptors in the brain has been mapped and rhesus monkeys share many of the same socio-cognitive behaviors with humans (Chang and Platt, 2013; Freeman et al., 2014). However, only a handful of studies have examined the prosocial effects of OT in nonhuman primates (Winslow and Insel, 1991; Smith et al., 2010; Saito and Nakamura, 2011; Simpson et al., 2014). Using an IN aerosolized (AE) administration procedure, Parr et al. (2013) reported that OT reduced monkeys' $(N=6)$ attention towards negative facial expressions, while increasing attention to direct vs. averted gaze faces. They concluded that INOT may reduce the negative, aversive, or threatening quality of social images which may, in turn, enable monkeys to engage in more prosocial behaviors and/or enhance social learning. Similarly, Ebitz et al. (2013) reported that IN-OT reduced social vigilance in rhesus monkeys, decreasing subjects' attentional bias to dominant faces, and diminishing the distraction caused by threatening facial expressions in a directed saccade task. Subject numbers for the tasks performed in this study ranged from 2-4. In a study directed more at social motivation and reward, Chang et al. (2012) reported that when monkeys $(N=2)$ were given the choice between rewarding themselves (with juice) compared to another monkey, they chose selfishly, an act not affected by IN-OT. However, if the choice was between rewarding another monkey vs. rewarding no one, IN-OT enhanced the subjects' choice to reward the other, suggesting that IN-OT enhanced social reward vicariously.

It was the goal of the current study to explore directly the influence of IN-OT on social reward in rhesus monkeys by modifying the experimental paradigm of Hurlemann et al. (2010). Monkeys were trained to perform an implicit visual matching task in which correct answers were rewarded with food, in addition to either an emotionally positive or negative social (facial expressions) or nonsocial image (familiar objects). We hypothesized that, like humans, IN-OT would enhance socially-reinforced learning in our monkeys and that this would be greater when the reinforcing images were emotionally positive compared to negative.

\section{METHODS}

\section{SUBJECTS}

Four adult male rhesus monkeys (aged 6-13 years) were used in these studies. All were born into large social groups and motherreared for the first year of life at the Yerkes Primate Center field station. All had been used in previous studies of face processing and one prior study of intranasal OT and social attention (Parr, 2011; Parr et al., 2013). All subjects were housed in same-sex pairs in the same colony room and were not food or water restricted for participation in these studies.

\section{TESTING PROCEDURE AND STIMULI}

Subjects were tested in their home cage using a touchscreen-based matching-to-sample (MTS) task. A custom panel containing a touchscreen monitor and opening for an automated feeder was inserted into the doorway of each subject's home cage. In brief, the MTS task first presented subjects with a 300 pixel stimulus (the sample) located centrally on the upper portion of the screen. After touching this stimulus, it disappeared from the screen and was followed by the presentation of two comparison images $(155 \times 155$ pixels $)$. If subjects contacted the correct comparison image, both were removed simultaneously from the screen and replaced by a centrally-located reward stimulus $(500 \times 500$ pixels). This remained on the screen for approximately $4 \mathrm{~s}$ during the automatic delivery of a small food-reward. The screen then returned to black for an intertrial interval (ITI) of $1 \mathrm{~s}$. After this, the next sample image appeared on the screen designating a new trial. If subjects selected the incorrect image, the screen immediately went black, there was no food reward, and the ITI was increased to $5 \mathrm{~s}$. All subjects had been trained on the basic MTS procedures prior to this study (Parr, 2011).

For the present study, subjects were presented with four different trials, each showing a unique combination of three different clip art images (the sample image plus two comparisons, per trial). The correct pair for each trial was chosen arbitrarily prior to testing and there was no obvious relationship between the matching pair of images in terms of content or shape. Sample images were presented in color and the comparison images in black and white to eliminate the potential use of color cue. To perform the task correctly, subjects simply had to remember which of the two comparison clip art images was rewarded and select it in subsequent trials. After the correct completion of each trial, subjects were presented with one of four "reinforcement" images, e.g., either positive or negative social or nonsocial 
image. The positive social image was a monkey play face and the positive nonsocial image was a picture of a banana. The negative social image was a monkey open mouth threat face and the negative nonsocial image was a picture of a cage lock. Figure 1 illustrates examples of each type of MTS trial showing the positive (Figure 1A) and negative (Figure 1B) reinforcement stimuli.
It was unknown how quickly subjects would learn the implicit matching task or when, if ever, ceiling effects would be achieved. In previous MTS studies, these same subjects routinely completed sessions containing up to 100 MTS trials without fatigue (Parr, 2011). Thus, in this experiment, subjects were presented with a total of 80 trials, representing 20 different presentations of each of the four clip art matching trials. These were presented
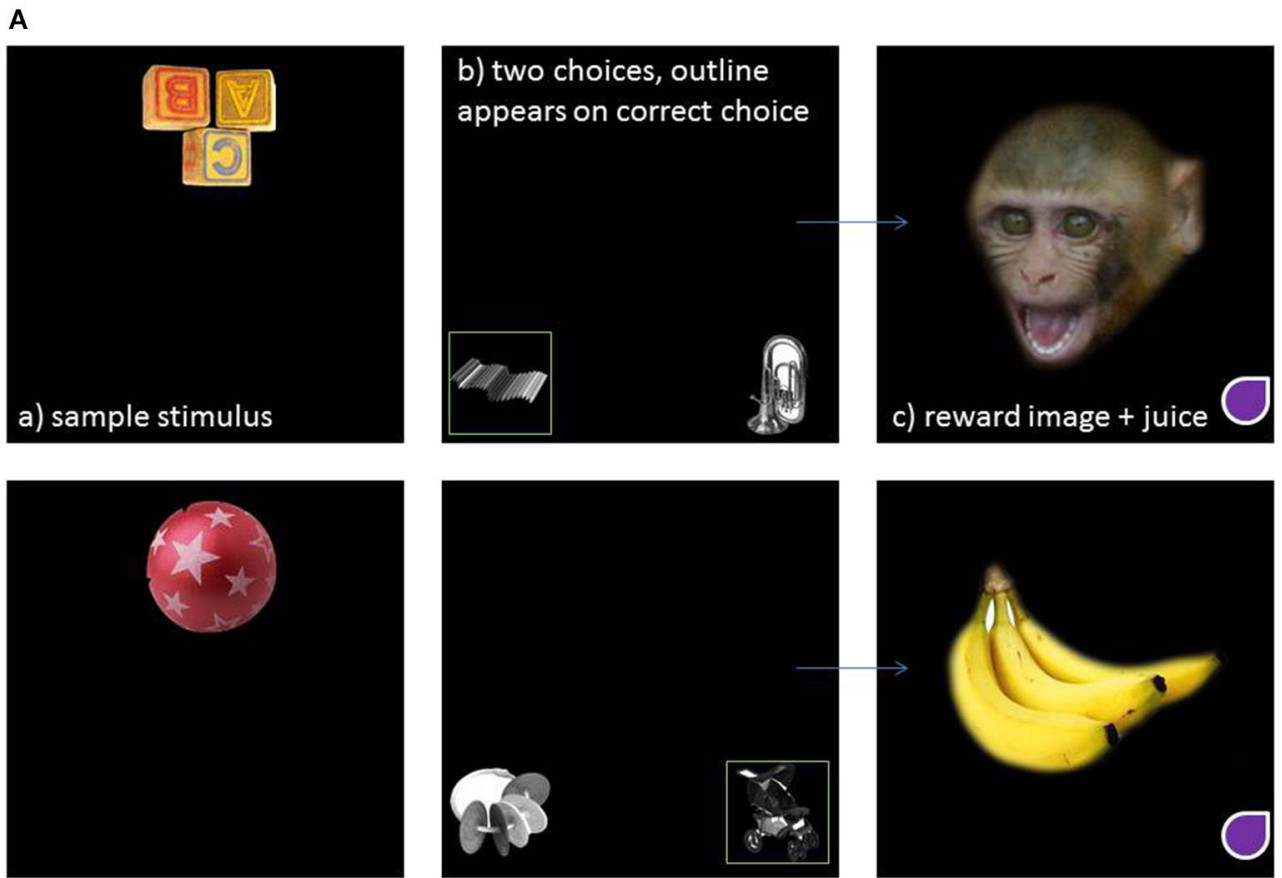

B
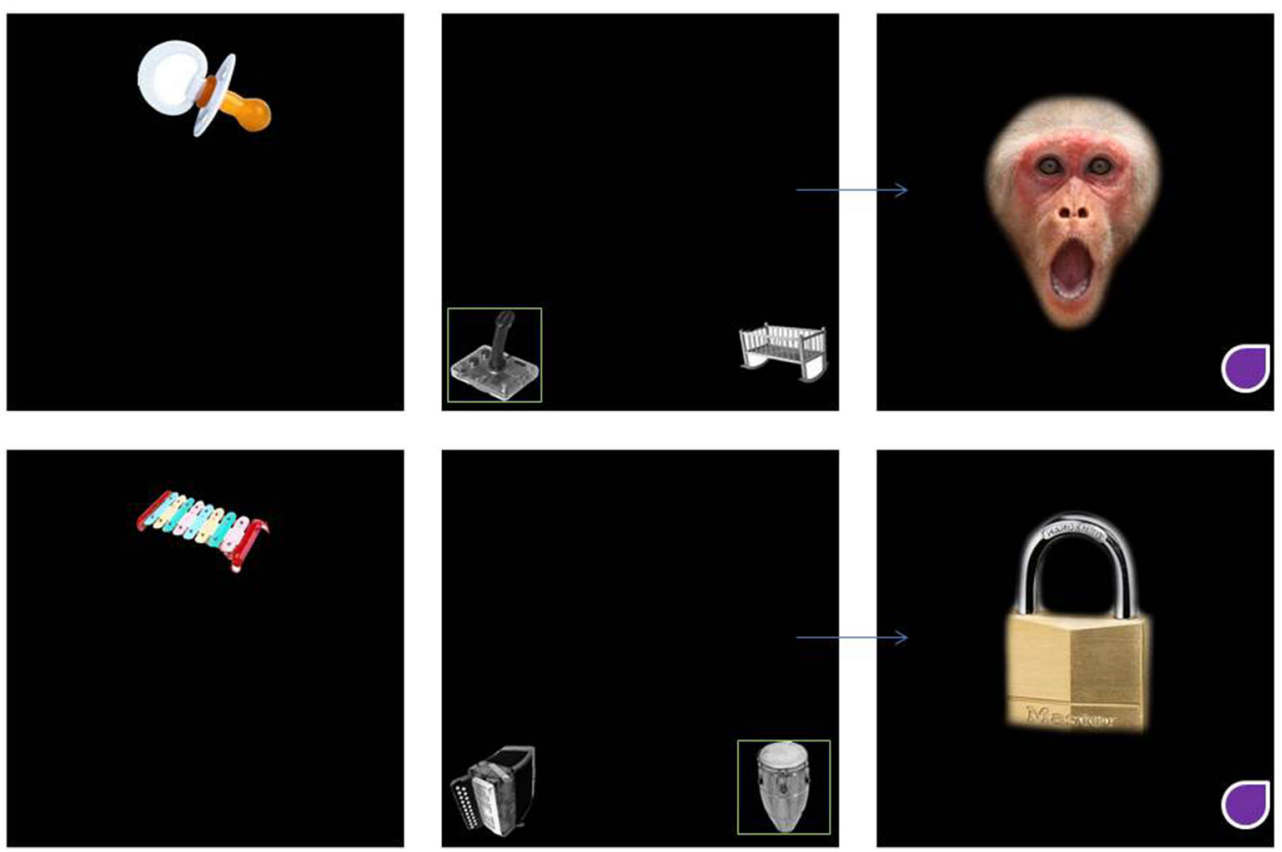

FIGURE 1 | An example of an implicit visual matching trial where the reinforcing stimuli are positive social and nonsocial (A) and negative social and nonsocial (B) 
in a pseudorandom, counterbalanced order. To assess learning, performance was averaged across 10, 8-trial blocks, totaling the 80 trial session. Two testing sessions were performed overall for each treatment condition (OT and P) and each contained novel stimuli.

\section{OXYTOCIN ADMINISTRATION PROCEDURE}

Prior to testing, subjects were administered either a 48IU dose of OT (Sigma \#06379, $1.71 \mu \mathrm{g} / \mathrm{IU}$ in a $2 \mathrm{ml}$ volume saline) or $2 \mathrm{ml}$ of saline placebo in aerosol form using a pediatric nebulizer. ${ }^{1}$ This method has been proven effective in delivering OT to the central nervous system (CNS) in rhesus monkeys (Chang et al., 2012; Parr et al., 2013; Modi et al., 2014). The same monkeys used here had been trained for this procedure for a previous study (Parr et al., 2013). In brief, each subject was placed in a specially designed cage that contained a clear Plexiglas panel to which the nebulizer was attached. Also inserted into this panel was a small tube through which a slow stream $(9 \mathrm{ml} / \mathrm{min})$ of fluid reward (diluted yogurt) was delivered. This encouraged subjects to maintain their face in a position over aerosol stream and breathe through their nose while sipping the fluid. Subjects were required to sip on this tube for 4 cumulative min within a $7 \mathrm{~min}$ window while $\mathrm{AE}$ saline was delivered just below the nose of the subject through the nebulizer tube. Given the broad range of doses and administration routes used in previous animal and human studies, no attempt was made to correct the dose for subjects' body weight (Guastella et al., 2013). Weights of subjects ranged from 7 to $15 \mathrm{~kg}$.

For each task, the order of treatment condition (placebo vs. OT) was counterbalanced across subjects for the two testing sessions. Half of the subjects were first tested in Placebo condition while the other half received the OT condition and this order was then reversed in session 2. Testing on each treatment condition was separated by a minimum of 7 days. Testing began $\sim 60 \mathrm{~min}$ after dosing, comparable to the peak of OT in CSF (Chang et al., 2012; Modi et al., 2014). During this time, subjects rested in their home cage. After $60 \mathrm{~min}$, a custom designed touchscreen panel was attached to the subject's home cage to administer the task. All procedures were approved by the Institutional Animal Care and Use Committee of Emory University and were in compliance with regulations governing the ethical treatment of animals.

\section{DATA ANALYSIS}

An initial repeated measures ANOVA was performed to determine whether performance differed between the two testing sessions. Performance was then analyzed using a full factorial repeated measures ANOVA that contained 2-levels of treatment condition (OT vs. P), 2-levels of social content (social vs. nonsocial), and 10 levels of trial block as the within-subject factors. Because treatment conditions were performed on different days, subsequent analyses compared the influence of social content (social vs. nonsocial) on performance ( $N=10$ trial blocks) separately for each treatment condition. A final repeated measures ANOVA was performed comparing the effect of emotional valence (positive vs. negative) and social content (social vs. nonsocial) on subjects'

\footnotetext{
${ }^{1}$ www.pari.com
}

performance. Follow-up analyses were performed using paired $t$ tests, where appropriate. Levels of significance were set at $p<0.05$ (two-tailed) and effect sizes are reported.

\section{RESULTS}

An initial repeated measures ANOVA revealed no main effect of testing session, $F_{(1,1)}=1.42, p=0.45$, so the data were combined and subsequent analyses were performed on the complete dataset.

A repeated measures ANOVA was first used to examine the effects of treatment condition (OT vs. P), social content (social vs. nonsocial), and trial block $(n=10)$ on subjects' task performance. This revealed main effects of social content, $F_{(1,3)}=29.10$, $p=0.012$, eta $^{2}=0.91$, and trial block, $F_{(9,27)}=2.80, p=0.018$, eta $^{2}=0.48$. Subjects performed significantly better on trials that were reinforced with social vs. nonsocial images (73\% vs. $52 \%$, respectively), and performance showed a significant improvement across the 10-trial blocks, linear: $F_{(1,3)}=12.15, p=0.04$, eta ${ }^{2}=$ 0.80 . There was also a significant interaction between treatment condition and social content, $F_{(1,3)}=11.49, p=0.043$, eta ${ }^{2}=$ 0.79. To further examine this interaction, a follow-up analysis compared performance on the social vs. nonsocial trials within each treatment condition. This revealed significant effects of socially-reinforced learning, e.g., improvements in performance after social vs. nonsocial reinforcement, for in both treatment conditions but this effect was considerably stronger for the OT, $t_{(3)}=6.26, p=0.008$, compared to Placebo, $t_{(3)}=3.78, p=0.032$, condition. Figures 2, 3 show the mean performance across trial blocks for the social and nonsocial trials in the Placebo and OT conditions, respectively.

The decision to give subjects 80 trials to assess sociallyreinforced learning was based on the need to provide a large enough number of trials to ensure that some learning would take place but also understanding that ceiling effects may occur, particularly towards the end of the testing session. Therefore, to assess differences in the rate of learning across the 80-trial sessions, we conducted a series of post-hoc analyses on the data presented in Figures 2, 3 that increased the number of trial

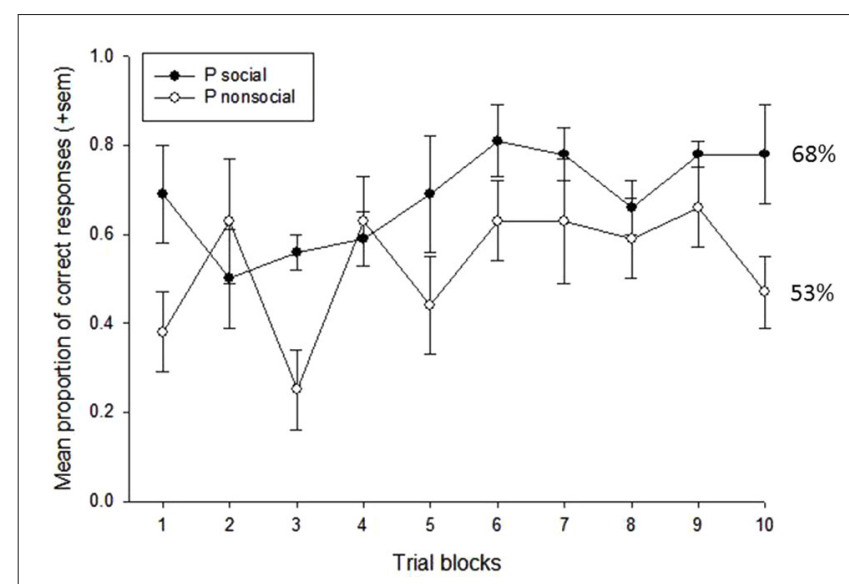

FIGURE 2 | Mean proportion of correct responses (+sem) for socially and non socially-reinforced trials in the Placebo condition. Overall performance (\%correct) is listed for each category. 
Table 1 | Results of post-hoc repeated measures ANOVAs focused on main effects of social condition and social condition by trial block interactions for increasing numbers of trial blocks.

\begin{tabular}{|c|c|c|c|c|c|c|}
\hline \multirow{2}{*}{$\begin{array}{l}\text { Placebo } \\
\text { Number of trial blocks }\end{array}$} & \multicolumn{3}{|c|}{ Main Effect: Social Content } & \multicolumn{3}{|c|}{ Social Content $\times$ Trial Block } \\
\hline & $F$-value (df) & $p$-value & eta $^{2}$ & $F$-value (df) & $p$-value & eta $^{2}$ \\
\hline 2 blocks & $1.42(1,3)$ & 0.319 & 0.32 & $4.2(1,3)$ & 0.13 & 0.58 \\
\hline 4 blocks & $11.44(1,3)$ & 0.043 & 0.79 & $3.09(3,9)$ & 0.083 & 0.51 \\
\hline 5 blocks & $7.25(1,3)$ & 0.074 & 0.71 & $2.36(4,12)$ & 0.113 & 0.44 \\
\hline 6 blocks & $9.45(1,3)$ & 0.054 & 0.76 & $1.93(5,15)$ & 0.148 & 0.39 \\
\hline 9 blocks & $9.52(1,3)$ & 0.054 & 0.76 & $1.58(8.24)$ & 0.183 & 0.35 \\
\hline \multirow{2}{*}{$\begin{array}{l}\text { OT } \\
\text { Number of trial blocks }\end{array}$} & \multicolumn{3}{|c|}{ Main Effect: Social Content } & \multicolumn{3}{|c|}{ Social Content $\times$ Trial Block } \\
\hline & F-value (df) & $p$-value & eta $^{2}$ & $F$-value (df) & $p$-value & eta $^{2}$ \\
\hline 2 blocks & $32.11(1,3)$ & 0.011 & 0.92 & $0.07(1,3)$ & 0.809 & 0.02 \\
\hline 3 blocks & $72.60(1,3)$ & 0.003 & 0.96 & $0.83(2,6)$ & 0.481 & 0.22 \\
\hline 8 blocks & $33.35(1,3)$ & 0.010 & 0.92 & $1.94(7,21)$ & 0.113 & 0.93 \\
\hline 9 blocks & $47.54(1,3)$ & 0.006 & 0.94 & $1.06(8,24)$ & 0.421 & 0.26 \\
\hline
\end{tabular}

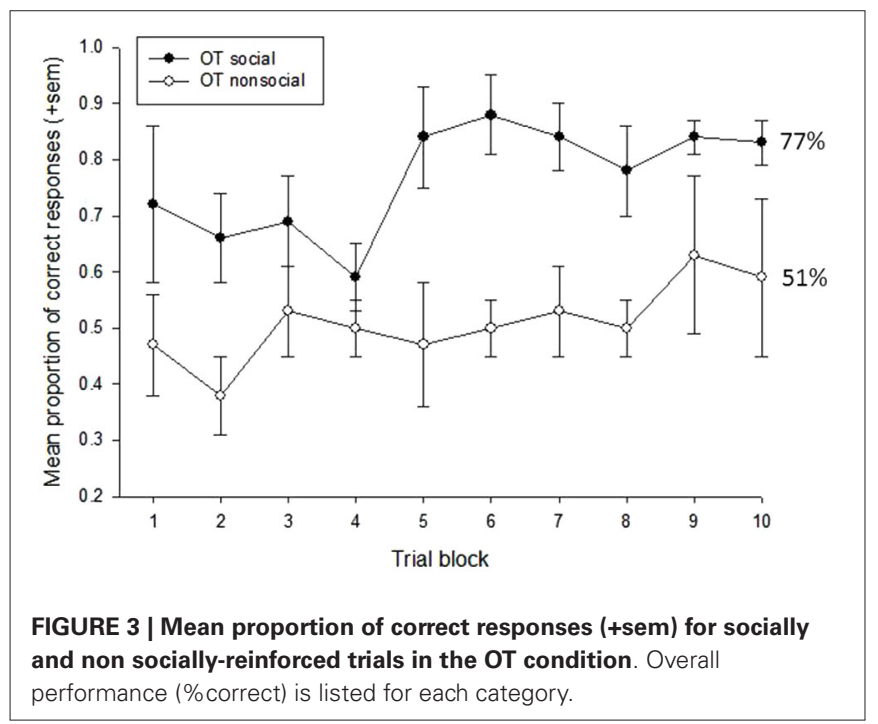

blocks in a stepwise manner to determine how many blocks would be needed before learning effects could be detected in each treatment condition. These results can be seen in Table 1. This revealed consistently stronger and sustained effects of treatment condition and treatment condition by trial block interactions for socially-reinforced trials in the OT compared to Placebo conditions.

Finally, to determine whether the emotional valence of the reinforcement stimuli (positive vs. negative) influenced subjects' performance, an additional repeated measures ANOVAs was performed for each treatment condition using social condition (social vs. nonsocial), valence (positive vs. negative) and trial block (10) as the within-subject factors. In the Placebo condition, there was a main effect of trial, $F_{(9,27)}=2.89, p<0.02$, eta ${ }^{2}=0.49$, showing that performance improved over time. No other main effect or interaction reached significance. In the OT condition, however, there were significant main effects of social condition, $F_{(1,2)}=35.27, p<0.03$, eta ${ }^{2}=0.95$, and stimulus valence, $F_{(1,2)}=$ 96.59, $p<0.01$, eta ${ }^{2}=0.98$. Performance was significantly better after social compared to nonsocial reinforcement (social $=74 \%$ vs. nonsocial $=48 \%$ ) and when the reinforcement images were emotionally positive compared to negative (positive $=66 \%$ vs. negative $=55 \%$ ).

\section{DISCUSSION}

This study is the first to demonstrate an enhancing effect of OT on socially-reinforced learning in a nonhuman primate, the rhesus monkey. The analyses revealed two main findings that were independent of treatment condition and confirm the validity of our experimental design. First, as expected, subjects' performance improved steadily over time, revealing significant effects of learning on a simple implicit, visual matching task within a single 80 -trial session. Demonstrating the ability of this task to elicit learning within a single trial block was extremely important since the effects of OT begin to degrade an hour after administration (Chang et al., 2012; Modi et al., 2014). Additionally, this task has high translational value as it was adapted from a study of socially-reinforced learning in humans (Hurlemann et al., 2010) and is highly amenable to repeated testing within subjects by simply changing the matching pairs of images. The second main finding was that subjects performed better after being reinforced with social stimuli (plus food) compared to nonsocial stimuli 
(plus food). In this experiment, social stimuli consisted of speciestypical facial expressions, play faces and open mouth threats, while nonsocial stimuli consisted of object categories that were familiar to the subjects, bananas and cage locks. It should be noted that the manner in which subjects were rewarded in this study differed from the procedures used in the original human study (Hurlemann et al., 2010). As stated earlier, the testing procedure for humans was to reinforce subjects with a positive social or nonsocial image when the response was correct and use negative social or nonsocial images when the responses were incorrect, thus providing a type of punishment. However, for the present study, food rewards were necessary to reinforce the monkeys' behavior and provide positive feedback for successful performance on the task. If the incorrect trials were not food rewarded, but rather followed by negative images, one could not dissociate whether any observed learning in the monkeys was due to the presence of the negative image, or the absence of the food. Thus, correct responses by the monkeys were always rewarded with food but additionally reinforced with an image, while incorrect responses were never followed by food or image presentation.

Importantly, this study also demonstrated that the effect of socially-reinforced learning was enhanced, subjects learned faster and performed better, after OT compared to placebo treatment. In the OT condition, performance was significantly better for the trials reinforced with social compared to nonsocial images across each of the 10 trial blocks, whereas this finding was much weaker and occurred across a smaller number of trial blocks in the placebo condition. Therefore, similar to humans (Hurlemann et al., 2010), these results confirm the ability of IN-OT to enhance socially-reinforced learning in rhesus monkeys. Future studies should examine these findings with greater detail using a variety of social and nonsocial images paying special attention to the salience of the images, in addition to their social content. It is possible that the social images used in the present study, e.g., facial expressions, elicited greater attention than the nonsocial images due to their overall salience. While this is interesting and the salience of facial expressions is undoubtedly tied to their social nature, it would be informative to delve further into the extent to which the OT-enhanced effects of learning reported here are really unique to social stimuli, or whether they could extend to other highly salient but nonsocial categories. Interestingly, the worst performance in this study occurred in the placebo condition for trials reinforced with the positive, nonsocial stimulus, e.g., the banana. While this is difficult to interpret, perhaps strong interest in this desirable food item produced a type of appetitive interference which inhibited any reinforcing effects on learning.

Finally, subjects' performance was also influenced by the emotional valence of the reinforcement images, positive or negative. There were no significant effects of stimulus valence or social content in the placebo condition. However, in the OT condition, subjects performance was significantly better when subjects' were reinforced with social compared to nonsocial images, e.g., socially-reinforced learning, and their performance was significantly better when the reinforcing images were emotionally positive compared to negative, but the interaction between social content and stimulus valence was not significant. Again, this result confirms the overall findings that OT enhances socially-reinforced learning and that positive social stimuli facilitate learning. In general, the valence effects found in monkeys are consistent with reports from human studies showing that IN-OT does not affect the perception of all social stimuli the same way, rather it can selectively affect the perception of positive or negative emotional stimuli, depending on the nature of the task (Domes et al., 2013).

There has been some debate about the general mechanisms behind the effects of OT on primate behavior, both in humans and animals (Churchland and Winkielman, 2012). OT, a large molecule peptide, is unable to cross the blood brain barrier if administered peripherally. However, an early study in humans showed that a related peptide, vasopressin, was able to achieve central penetrance after IN administration (Born et al., 2002), possibly through a "direct transport route" where OT obtains rapid access to the CNS through numerous perineuronal spaces in the nasal cavity where it can then enter the CSF (Veening and Olivier, 2013). Moreover, a recent study has now reported that IN-OT administration in humans does increase central concentrations of OT (Striepens et al., 2013). However, the procedure for administering IN-OT to humans is very precise and tightly controlled. Subjects are instructed to self-administer the OT spray by closing one nostril and deeply inhaling (sniffing) as they spray the OT into the open nostril. A typical dose of OT nasal spray (Syntocinon, Novartis) is 24IU which corresponds to 6, 4IU squirts, three given to each nostril. Nonhuman primates cannot easily be trained to follow these procedures that require them to actively sniff as the spray is delivered by a human experimenter. Instead, primate researchers have adopted a modified IN procedure using a passive breathing method where OT is delivered in aerosol form using a nebulizer. Chang et al. (2012) were the first to demonstrate that an AE IN route is effective in increasing the central penetrance of OT in rhesus monkeys. Our group has further compared several methods of OT administration in rhesus monkeys, including IN, AE and intravenous (IV) and found that only the AE was effective in elevating central OT levels (Modi et al., 2014). Additionally, these studies also report large and long-lasting elevations of peripheral OT in blood after all administration routes and thus the behavioral effects of IN or AE OT could also be influenced by positive feedback from peripheral OT receptors (Neumann et al., 2013; Veening and Olivier, 2013). The importance of this discussion is that working with young human infants presents many of the same methodological challenges as monkeys, thus the AE method may actually represent the most effective and least stressful method for administering IN-OT in this population.

The results of the present study have important implications for the development of treatments for social deficits, such as those observed in individuals with ASD. According to the social motivation theory of autism, individuals with ASD fail to find social stimuli rewarding, reducing their attention and/or willingness to interact with others (Chevallier et al., 2012). This lack of social interest can have long lasting effects on the developing social brain and may contribute substantially to the social communication deficits that comprise a core symptom of ASD. Neuroimaging 
studies during an implicit learning task in which subjects were reinforced with social (faces) vs. nonsocial (money) rewards, found greater activation in the brain's reward circuitry, the anterior cingulate cortex, ventral prefrontal cortex, and striatum, in response to the social reward condition in typical compared to individuals with ASD. This study suggests that neural reward circuitry may be dysregulated in individuals with ASD compared to typicals, supporting the social motivation theory (Scott-Van Zeeland et al., 2010). Interestingly, differences in the activation of reward circuitry between ASD and control individuals were only found using a between-group analysis. Within the ASD group, this study did not find differences in reward circuitry in response to social vs. nonsocial reward and, interestingly, studies have reported typical reward circuitry activation in individuals with ASD in response to their preferred objects, e.g., those valued by the subjects like trains and electronics (Dichter et al., 2012). This finding has important treatment-related implications as it suggests that the general reward circuitry of the ASD brain may not be completely dysregulated, rather it may not be activated by typical social rewards, like faces. Considering these findings, it may be possible to use IN-OT in combination with behavioral therapies to increase the rewarding quality of typical social stimuli, like eyes and faces, in children with autism, or at risk for developing ASD, to advance social learning and perhaps alleviate some of the more long-lasting deficits observed in social cognition.

\section{ACKNOWLEDGMENTS}

This project was funded by R01MH068791 and K02MH096084 to Lisa A. Parr. Additional support was provided by the National Center for Research Resources P51RR000165 to the Yerkes National Primate Research Center, currently the Office of Research Infrastructure Programs/OD P51OD011132. Thanks to Erin Siebert and Lauren Murphy for animal assistance, and Dr. Hasse Walum for comments on a previous version of this manuscript.

\section{REFERENCES}

Andari, E., Duhamel, J. R., Zalla, T., Herbrecht, E., Leboyer, M., and Sirigu, A. (2010). Promoting social behavior with oxytocin in high-functioning autism spectrum disorders. Proc. Natl. Acad. Sci. U S A 107, 4389-4394. doi: 10. 1073/pnas.0910249107

Born, J., Lange, T., Kern, W., McGregor, G. P., Bickel, U., and Fehm, H. L. (2002). Sniffing neuropeptides: a transnasal approach to the human brain. Nat. Neurosci. 5, 514-516. doi: 10.1038/nn0602-849

Chang, S. W., Barter, J. W., Ebitz, R. B., Watson, K. K., and Platt, M. L. (2012). Inhaled oxytocin amplifies both vicarious reinforcement and self reinforcement in rhesus macaques (Macaca mulatta). Proc. Natl. Acad. Sci. US A 109, 959-964. doi: 10.1073/pnas.1114621109

Chang, S. W., and Platt, M. L. (2013). Oxytocin and social cognition in rhesus macaques: implications for understanding and treating human psychopathology. Brain Res. doi: 10.1016/j.brainres.2013.11.006. [Epub ahead of print].

Chevallier, C., Kohls, G., Troiani, V., Brodkin, E. S., and Schultz, R. T. (2012). The social motivation theory of autism. Trends Cogn. Sci. 16, 231-239. doi: 10.1016/j. tics.2012.02.007

Churchland, P. S., and Winkielman, P. (2012). Modulating social behavior with oxytocin: how does it work? What does it mean? Horm. Behav. 61, 392-399. doi: 10.1016/j.yhbeh.2011.12.003

Dawson, G., Meltzoff, A. N., Osterling, J., Rinaldi, J., and Brown, E. (1998). Children with autism fail to orient to naturally occurring social stimuli. J. Autism Dev. Disord. 28, 479-485. doi: 10.1023/A:1026043926488
Delmonte, S., Balsters, J. H., Mcgrath, J., Fitzgerald, J., Brennan, S., Fagan, A. J., et al. (2012). Social and monetary reward processing in autism spectrum disorders. Mol. Autism 3:7. doi: 10.1186/2040-2392-3-7

Dichter, G. S., Felder, J. N., Green, S. R., Rittenberg, A. M., Sasson, N. J., and Bodfish, J. W. (2012). Reward circuitry function in autism spectrum disorders. Soc. Cogn. Affect. Neurosci. 7, 160-172. doi: 10.1093/scan/nsq095

Domes, G., Heinrichs, M., Glascher, J., Buchel, C., Braus, D. F., and Herpertz, S. C. (2007a). Oxytocin attenuates amygdala responses to emotional faces regardless of valence. Biol. Psychiatry 62, 1187-1190. doi: 10.1016/j.biopsych.2007. 03.025

Domes, G., Heinrichs, M., Kumbier, E., Grossmann, A., Hauenstein, K., and Herpertz, S. C. (2013). Effects of intranasal oxytocin on the neural basis of face processing in autism spectrum disorder. Biol. Psychiatry 74, 164-171. doi: 10. 1016/j.biopsych.2013.02.007

Domes, G., Heinrichs, M., Michel, A., Berger, C., and Herpertz, S. C. (2007b). Oxytocin improves "mind-reading" in humans. Biol. Psychiatry 61, 731-733. doi: 10.1016/j.biopsych.2006.07.015

Ebitz, R. B., Watson, K. K., and Platt, M. L. (2013). Oxytocin blunts social vigilance in the rhesus macaque. Proc. Natl. Acad. Sci. U S A 110, 11630-11635. doi: 10. 1073/pnas. 1305230110

Fischer-Shofty, M., Shamay-Tsoory, S. G., Harari, H., and Levkovitz, Y. (2010). The effect of intranasal administration of oxytocin on fear recognition. Neuropsychologia 48, 179-184. doi: 10.1016/j.neuropsychologia.2009.09.003

Freeman, S. M., Inoue, K., Smith, A. L., Goodman, M. M., and Young, L. J. (2014). The neuroanatomical distribution of oxytocin receptor binding and mRNA in the male rhesus macaque (Macaca mulatta). Psychoneuroendocrinology 45, 128-141. doi: 10.1016/j.psyneuen.2014.03.023

Gamer, M., Zurowski, B., and Buchel, C. (2010). Different amygdala subregions mediate valence-related and attentional effects of oxytocin in humans. Proc. Natl. Acad. Sci. U S A 107, 9400-9405. doi: 10.1073/pnas.1000985107

Guastella, A. J., Einfeld, S. L., Gray, K. M., Rinehart, N. J., Tonge, B. J., Lambert, T. J., et al. (2010). Intranasal oxytocin improves emotion recognition for youth with autism spectrum disorders. Biol. Psychiatry 67, 692-694. doi: 10.1016/j. biopsych.2009.09.020

Guastella, A. J., Hickie, I. B., McGuinness, M. M., Otis, M., Woods, E. A., Disinger, H. M., et al. (2013). Recommendations for the standardisation of oxytocin nasal administration and guidelines for its reporting in human research. Psychoneuroendocrinology 38, 612-625. doi: 10.1016/j.psyneuen.2012. 11.019

Guastella, A. J., and MacLeod, C. (2012). A critical review of the influence of oxytocin nasal spray on social cognition in humans: evidence and future directions. Horm. Behav. 61, 410-418. doi: 10.1016/j.yhbeh.2012. 01.002

Guastella, A. J., Mitchell, P. B., and Dadds, M. R. (2008). Oxytocin increases gaze to the eye region of human faces. Biol. Psychiatry 63, 3-5. doi: 10.1016/j.biopsych. 2007.06.026

Hollander, E., Novotny, S., Hanratty, M., Yaffe, R., DeCaria, C. M., Aronowitz, B. R., et al. (2003). Oxytocin infusion reduces repetitive behaviors in adults with autistic and Asperger's disorders. Neuropsychopharmacology 28, 193-198. doi: 10.1038/sj.npp.1300021

Hurlemann, R., Patin, A., Onur, O. A., Cohen, M. X., Baumgartner, T., Metzler, S., et al. (2010). Oxytocin enhances amygdala-dependent, socially reinforced learning and emotional empathy in humans. J. Neurosci. 30, 4999-5007. doi: 10. 1523/JNEUROSCI.5538-09.2010

Modi, M. E., Connor-Stroud, F., Landgraf, R., Young, L. J., and Parr, L. A. (2014). Aerosolized oxytocin increases cerebrospinal fluid oxytocin in rhesus macaques. Psychoneuroendocrinology 45, 49-57. doi: 10.1016/j.psyneuen.2014.02.011

Modi, M. E., and Young, L. J. (2012). The oxytocin system in drug discovery for autism: animal models and novel therapeutic strategies. Horm. Behav. 61, 340-350. doi: 10.1016/j.yhbeh.2011.12.010

Neumann, I. D., Maloumby, R., Beiderbeck, D. I., Lukas, M., and Landgraf, R. (2013). Increased brain and plasma oxytocin after nasal and peripheral administration in rats and mice. Psychoneuroendocrinology 38, 1985-1993. doi: 10. 1016/j.psyneuen.2013.03.003

Parr, L. A. (2011). The evolution of face processing in primates. Philos. Trans. R. Soc. Lond. B Biol. Sci. 366, 1764-1777. doi: 10.1098/rstb.2010.0358

Parr, L. A., Modi, M., Siebert, E., and Young, L. J. (2013). Intranasal oxytocin selectively attenuates rhesus monkeys' attention to negative facial expressions. 
Psychoneuroendocrinology 38, 1748-1756. doi: 10.1016/j.psyneuen.2013. 02.011

Preti, A., Melis, M., Siddi, S., Vellante, M., Doneddu, G., and Fadda, R. (2014). Oxytocin and autism: a systematic review of randomized controlled trials. J. Child Adolesc. Psychopharmacol. 24, 54-68. doi: 10.1089/cap.2013. 0040

Rimmele, U., Hediger, K., Heinrichs, M., and Klaver, P. (2009). Oxytocin makes a face in memory familiar. J. Neurosci. 29, 38-42. doi: 10.1523/JNEUROSCI.426008.2009

Ross, H. E., and Young, L. J. (2009). Oxytocin and the neural mechanisms regulating social cognition and affiliative behavior. Front. Neuroendocrinol. 30 , 534-547. doi: 10.1016/j.yfrne.2009.05.004

Saito, A., and Nakamura, K. (2011). Oxytocin changes primate paternal tolerance to offspring in food transfer. J. Comp. Physiol. A Neuroethol. Sens. Neural Behav. Physiol. 197, 329-337. doi: 10.1007/s00359-010-0617-2

Savaskan, E., Ehrhardt, R., Schulz, A., Walter, M., and Schachinger, H. (2008). Post-learning intranasal oxytocin modulates human memory for facial identity. Psychoneuroendocrinology 33, 368-374. doi: 10.1016/j.psyneuen.2007. 12.004

Scott-Van Zeeland, A. A., Dapretto, M., Ghahremani, D. G., Poldrack, R. A., and Bookheimer, S. Y. (2010). Reward processing in autism. Autism Res. 3, 53-67. doi: 10.1002/aur.122

Simpson, E. A., Sclafani, V., Paukner, A., Hamel, A. F., Novak, M. A., Meyer, J. S., et al. (2014). Inhaled oxytocin increases positive social behaviors in newborn macaques. Proc. Natl. Acad. Sci. U S A 111, 6922-6927. doi: 10.1073/pnas. 1402471111

Smith, A. S., Agmo, A., Birnie, A. K., and French, J. A. (2010). Manipulation of the oxytocin system alters social behavior and attraction in pair-bonding primates, Callithrix penicillata. Horm. Behav. 57, 255-262. doi: 10.1016/j.yhbeh.2009. 12.004
Strathearn, L., Fonagy, P., Amico, J., and Montague, P. R. (2009). Adult attachment predicts maternal brain and oxytocin response to infant cues. Neuropsychopharmacology 34, 2655-2666. doi: 10.1038/npp.2009.103

Striepens, N., Kendrick, K. M., Hanking, V., Landgraf, R., Wullner, U., Maier, W., et al. (2013). Elevated cerebrospinal fluid and blood concentrations of oxytocin following its intranasal administration in humans. Sci. Rep. 3:3440. doi: 10. 1038/srep03440

Veening, J. G., and Olivier, B. (2013). Intranasal administration of oxytocin: behavioral and clinical effects, a review. Neurosci. Biobehav. Rev. 37, 1445-1465. doi: 10.1016/j.neubiorev.2013.04.012

Winslow, J. T., and Insel, T. R. (1991). Social status in pairs of male squirrel monkeys determines the behavioral response to central oxytocin administration. J. Neurosci. 11, 2032-2038.

Conflict of Interest Statement: The Guest Associate Editor Alaine Keebaugh declares that, despite being affiliated to the same institution as author Lisa A. Parr, the review process was handled objectively and no conflict of interest exists. The author declares that the research was conducted in the absence of any commercial or financial relationships that could be construed as a potential conflict of interest.

Received: 29 May 2014; accepted: 29 July 2014; published online: 23 September 2014. Citation: Parr LA (2014) Intranasal oxytocin enhances socially-reinforced learning in rhesus monkeys. Front. Behav. Neurosci. 8:278. doi: 10.3389/fnbeh.2014.00278 This article was submitted to the journal Frontiers in Behavioral Neuroscience. Copyright $\odot 2014$ Parr. This is an open-access article distributed under the terms of the Creative Commons Attribution License (CC BY). The use, distribution or reproduction in other forums is permitted, provided the original author(s) or licensor are credited and that the original publication in this journal is cited, in accordance with accepted academic practice. No use, distribution or reproduction is permitted which does not comply with these terms. 